\title{
New molecular classification of large cell neuroendocrine carcinoma and small cell lung carcinoma with potential therapeutic impacts
}

\author{
Sylvie Lantuejoul ${ }^{1,2}$, Lynnette Fernandez-Cuesta ${ }^{3}$, Francesca Damiola ${ }^{1}$, Nicolas Girard ${ }^{4}$, Anne McLeer ${ }^{2,5}$ \\ ${ }^{1}$ Department of Biopathology, Pathology Research Platform- Synergie Lyon Cancer- CRCL, Centre Léon Bérard Unicancer, Lyon, France; \\ ${ }^{2}$ Université Grenoble Alpes, Grenoble, France; ${ }^{3}$ Section of Genetics, International Agency for Research on Cancer (IARC-WHO), Lyon, France; \\ ${ }^{4}$ Institut Curie, Institut du Thorax Curie Montsouris, Paris, France; ${ }^{5}$ Department of Pathology and Cancer Molecular Genetics Platform, CHU \\ Grenoble Alpes, Grenoble, France \\ Contributions: (I) Conception and design: All authors; (II) Administrative support: All authors; (III) Provision of study materials or patients: All \\ authors; (IV) Collection and assembly of data: All authors; (V) Data analysis and interpretation: All authors; (VI) Manuscript writing: All authors; (VII) \\ Final approval of manuscript: All authors. \\ Correspondence to: Sylvie Lantuejoul, MD, PhD. Département de Biopathologie, Plateforme Anapath Recherche Synergie Lyon Cancer-Est CRCL, \\ Centre de Lutte Contre le Cancer UNICANCER Léon Bérard, 28 rue Laënnec, 69008 Lyon, France. Email: sylvie.lantuejoul@lyon.unicancer.fr.
}

\begin{abstract}
Large cell neuroendocrine carcinoma (LCNECs) and small cell lung carcinomas (SCLCs) are high-grade neuroendocrine carcinomas of the lung with very aggressive behavior and poor prognosis. Their histological classification as well as their therapeutic management has not changed much in recent years, but genomic and transcriptomic analyses have revealed different molecular subtypes raising hopes for more personalized treatment. Indeed, four subtypes of SCLCs have been recently described, SCLC-A driven by the master gene ASCL1, SCLC-N driven by NEUROD1, SCLC-Y by YAP1 and SCLC-P by POU2F3. Whereas SCLC standard of care is based on concurrent chemoradiation for limited stages and on chemotherapy alone or chemotherapy combined with anti-PD-L1 checkpoint inhibitors for extensive stage SCLC, SCLC-A variants could benefit from DLL3 or BCL2 inhibitors, and SCLC-N variants from Aurora kinase inhibitors combined with chemotherapy, or PI3K/mTOR or HSP90 inhibitors. In addition, a new SCLC variant (SCLC-IM) with high-expression of immune checkpoints has been also reported, which could benefit from immunotherapies. PARP inhibitors also gave promising results in combination with chemotherapy in a subset of SCLCs. Regarding LCNECs, they represent a heterogeneous group of tumors, some of them exhibiting mutations also found in SCLC but with a pattern of expression of NSCLC, while others harbor mutations also found in NSCLC but with a pattern of expression of SCLC, questioning their clinical management as NSCLCs or SCLCs. Overall, we are probably entering a new area, which, if personalized treatments are effective, will also lead to the implementation in practice of molecular testing or biomarkers detection for the selection of patients who can benefit from them.
\end{abstract}

Keywords: Lung cancer; neuroendocrine; high-grade; molecular; classification

Submitted Feb 16, 2020. Accepted for publication May 12, 2020.

doi: $10.21037 /$ tlcr-20-269

View this article at: http://dx.doi.org/10.21037/tlcr-20-269

\section{Introduction}

Lung neuroendocrine (NE) neoplasms account for approximately $20 \%$ of all lung cancers. They can be subdivided into low- and intermediate-grade NE tumors represented by typical and atypical carcinoids, and high-grade NE carcinomas represented by large cell neuroendocrine carcinoma (LCNEC) and small cell lung carcinoma (SCLC). LCNEC and SCLC account for $3 \%$ 
and $13-15 \%$ of all lung cancers, respectively. In recent years, the incidence of SCLC has slightly decreased, whereas that of LCNEC has discreetly raised $(1,2)$. The vast majority of LCNEC and SCLC patients are heavy smokers around 65 years of age, with metastatic disease at diagnosis. Both carcinoma types are very aggressive, with 5-year overall survival rates below 15-25\% (LCNEC) and 5\% (SCLC) for extensive stages (2,3). However, the therapeutic management of NSCLCs and SCLCs has not changed much in recent years, favoring for SCLCs platinum and etoposide-based chemotherapy and for LCNECs therapies close enough to that of NSCLCs.

However, whereas the histological classifications were unchanged until recently, several publications have dismembered LCNECs and SCLCs at the genomic and transcriptomic levels, leading to the identification of new molecular subtypes. The LCNEC group is heterogeneous, constituted both by tumors that harbor mutations that can be found in SCLC but with an expression pattern typical of NSCLC, and by tumors carrying typical NSCLC mutations but with a pattern of expression of SCLC, questioning their clinical management as NSCLCs or SCLCs. Regarding SCLCs, different "variants" have been successively described besides the predominant "classic" subtype, based on morphology, immunophenotype and expression profiles, and this distinction could allow for more personalized treatments.

\section{SCLC}

\section{Morphological classification}

According to the 2015 classification of lung tumors $(3,4)$, SCLCs are defined as malignant carcinomas with NE characteristics. They belong to the group of neuroendocrine neoplasms (NENs) of the lung and are the most aggressive NENs with the worst prognosis. Since the 2004 WHO classification (5), only pure SCLC and combined SCLC have been recognized. On histopathological examination, pure SCLC is composed of a dense proliferation of small tumor cells, arranged in sheets or ribbons, with extensive areas of necrosis. Less frequently, NE (organoid) features such as rosettes, palisades and nests may be found. At high power fields, tumor cells are round to spindle-shaped; they usually measure less than the size of 3 resting lymphocytes and have a sparse cytoplasm. The chromatin is finely granular, with a typical salt and pepper appearance and with inconspicuous nucleoli. Nuclear molding is common as well as apoptotic bodies and nuclear debris known as the Azzopardi effect. Mitoses are also numerous, with an average number of 80 per $2 \mathrm{~mm}^{2}$ area. These features are most evident in surgical specimens where tumor cells appear larger with a distinct cytoplasm and a focal vesicular chromatin (6). Combined SCLCs account for $10 \%$ to $25 \%$ of all SCLC and are defined by a mixture of pure SCLC and of adenocarcinoma, squamous cell, large cell or sarcomatoid (spindle or giant cells) carcinoma $(4,5)$, whatever the amount of NSCLC component. In contrast, for combined LCNEC and SCLC, a minimum of $10 \%$ of LCNEC is required. Combined SCLCs are more frequently diagnosed on widely sampled surgical specimens in comparison to biopsies $(7,8)$. Combined SCLC and pure SCLC share the same epidemiology and clinical presentation, even if combined SCLC tends to be more peripheral and could harbor a worse prognosis, possibly due to relative resistance of nonSCLC components to SCLC standard chemotherapy (9). Interestingly, an intermediate variant of SCLC was present in the 1981 WHO classification (10) and was defined by larger cells with distinct cell borders, a moderate amount of eosinophilic cytoplasm, and a single nucleus with a prominent central nucleolus and paranucleolar chromatin clearing (11). This intermediate subtype of SCLC was then removed from the subsequent classifications, the presence of larger cells being attributed to the larger size of the samples, with no significance per se.

\section{Immunophenotype}

Immunohistochemistry can be of great help to assert the diagnosis of SCLC, particularly on small biopsies when tumor cells are crushed. The vast majority of SCLCs diffusely express Achaete-Scute Family BHLH Transcription Factor 1 (ASCL1, also called hASH1) $(12,13)$ as well as CD56 (NCAM1) with a membrane staining $(14,15)$. In contrast, synaptic vesicle protein Synatophysin and dense-core associated protein Chromogranin A cytoplasmic stainings are observed in $54 \%$ and in $37 \%$ of the cases, respectively, and can be focal $(3,4,16-19)$. In addition, thyroid transcription factor 1 (TTF1) is expressed in $90 \%$ of SCLCs (20). However, $10 \%$ to $25 \%$ of SCLCs could lose neuroendocrine markers $(4,21)$ and this absence seems to be a characteristic of the intermediate variant described by Gazdar et al. in 1985 (11). In addition, some of the SCLCs with negative neuroendocrine markers have also been shown to lose cytokeratin and express vimentin. P16 nuclear staining is observed in almost all SCLCs, unlike 
$\mathrm{RB} 1$, which is assumed to be absent $(22,23)$. Regarding pathogenesis, SCLCs were initially supposed to arise from normal bronchial NE Kulchintsky-type cells, involved in lung growth and differentiation through the activation of airway chemoreceptors (24). It is now believed, based on genomic profiling studies, that SCLC could derive from multipotent precursor cells common to $\mathrm{NE}$ and nonNE pulmonary neoplasms. Indeed, Sutherland et al. (25) showed in transgenic mice that, while NE cells serve as the prevalent cell of origin of SCLC, SCLC can also arise, albeit with a lesser penetrance, from alveolar type II cells in which $\operatorname{Tr} p 53$ and $R b 1$ have been inactivated. The authors proposed that the loss of $R b 1$, a critical controller of NE differentiation, could be a mechanism by which, under specific conditions, a subset of progenitor-like alveolar type II cells could differentiate toward a more NE celllike state. These findings are in accordance with studies of treatment-resistant EGFR-mutated adenocarcinomas which have undergone a histological transformation to SCLC $(26,27)$. In these cases, small-cell tumours were identified by morphology and positive immunohistochemical staining for synaptophysin, chromogranin, or NCAM, together with a loss of RB1, and genomic sequencing showed that these transformed SCLC tumours retained the original EGFRactivating mutation (26). Furthermore, in transgenic mice, Lin $\mathrm{C}$ et al showed that expression of the EGFR exon 21 L858R mutation under the control of the SPC promoter leads to tumorigenesis (28). Alveolar type II cells could thus serve as the cell of origin of SCLC as well as EGFR-mutant adenocarcinoma, depending on the molecular alterations in presence, as EGFR TKI-resistant SCLCs have been shown to branch out early from the adenocarcinoma clones that harbor completely inactivated $R B 1$ and TP53 (27).

Of note, to date, no preneoplastic lesions have been identified for LCNEC or SCLC in humans, but preneoplastic neuroendocrine cells have been isolated in a mouse model of SCLC (29). Especially, no transitions from well-differentiated neuroendocrine tumors to SCLC have been described.

\section{Molecular classification: identification of SCLC subtypes}

Genome and transcriptome analyses on nearly 200 resected SCLCs (30-32) unveiled a high load of somatic mutations (8.62 nonsynonymous mutations per megabase). These molecular studies identified bi-allelic inactivation of TP53 and $R B 1$ in nearly all the tumors analyzed, mostly through mutations, loss of heterozygosity ( $\mathrm{LOH})$, or inactivating rearrangements. Inactivating mutations in $\mathrm{NOTCH}$ genes, which lead to the inactivation of HES1, an antagonist of ASCL1, were found in $25 \%$ of SCLC tumors. The transcription factors SOX2 and the MYC family of proteins were also frequently altered in SCLC: $S R Y$-box 2 (SOX2) was amplified in $27 \%$ of SCLC cases and MYC genes were altered through copy number amplification (9\% MYCL1, 4\% MYCN, and 6\% MYC) and fusion transcripts (RLF$M Y C L 1)$. In addition, recurrent mutations in chromatin remodeling genes such as $C R E B B P, E P 300$, as well as PTEN, SLIT2 or EPHA7 mutations or FGFR1 amplifications have also been described.

Two main key lineage-specific factors, ASCL1 and NEUROD1, responsible for neuroendocrine differentiation are reactivated in SCLC (33). ASCL1 activates NE differentiation, and regulates stemness, cell cycle progression, and mitosis. ASCL1 expression is limited to dormant progenitor pulmonary NE cells in mature lung but its reactivation in $\mathrm{NE}$ carcinomas maintains tumor development and survival. Its targets are MYCL1, RET, SOX2, BCL2 and nuclear factor I B $(N F I B)$ oncogenes, as well as NOTCH ligand DLL3. Of note, NKX2-1 (also called TTF1) gene expression is also positively regulated by ASCL1 (34) as well as BRN2 (brain-2; also known as POU3F2) (35). In contrast, NEUROD1 promotes neurogenic differentiation of cells during development and malignant behavior in SCLC cell lines. It targets $M Y C$ (36) and oncogenic $\mathrm{Myc}^{\mathrm{T} 58 \mathrm{~A}}$ promotes the development of slow growing NE tumors (37). Of note, both ASCL1-high and NEUROD1-high SCLCs express insulinoma associated protein 1 (INSM1), which is a driver of NE differentiation in many organs and tissues. Extensive transcriptional analyses performed in human tumors, genetically engineered mouse models (GEMMs) and patient-derived xenografts (PDXs) $(32,37,38)$ have revealed differential activation of these regulatory pathways. They led to the identification beside the classical SCLCs expressing high levels of ASCL1, of SCLC variants either expressing more NEUROD1 than ASCL1 or neither one (11,36,37,39-41). A certain plasticity has been reported between the different subtypes of SCLCs, with variants more frequently observed in tumors recurring after initial response to therapy (42). The dual ASCL1- and NEUROD1-negative variants have no NE phenotype and express RE1 silencing transcription factor (REST). They harbor an epithelial to mesenchymal transition (EMT) profile and activate the NOTCH, HIPPO and TGF $\beta$ pathways (40). These nonNE NOTCH-active SCLCs are probably slow growing tumors, but could be relatively chemoresistant in in vitro 
studies $(43,44)$. They are driven either by YAP1 or POU2F3 master genes $(32,45,46)$ with the current distinction of four subtypes of SCLCs, each of them mainly driven by a specific gene: SCLC-A, driven by ASCL1, SCLC-N by NEUROD1, SCLC-Y by YAP1 and SCLC-P by POU2F3 (47). YAP1 is a regulator of transcription activated by the HIPPO growth signaling pathway, and SCLC-Y tumors are RB1-positive by immunohistochemistry, which is rarely observed in SCLC (45), but they are enriched for CCND1 amplification and $C D K N 2 A$ inactivation, which result in RB1 inactivation and cell-cycle control defects (48). POU2F3 is a master regulator of the NE low SCLC subtype, involving IFGR1 pathway to increase cell proliferation. Interestingly, it has been proposed that SCLC-P tumors could arise from chemosensory tuft cells (46). Recently, Gay et al. identified by non-negative matrix factorization (NMF) analysis of RNAseq in a series of 81 resected SCLC tumor samples and 62 SCLC cell lines, a cluster called SCLC-IM, composed of "mesenchymal" tumors which lose cytokeratin and express vimentin, are NE markers negative and highly express immune checkpoints, STING-related genes, and inflammatory markers (49). This SCLC variant could be more sensitive to immune checkpoint inhibitors, which is of particular interest given the fact that the vast majority of SCLCs are considered as cold tumors. Indeed, they exhibit low levels of CD3, CD8 and CD20 tumor-infiltrating lymphocytes (TILs), low ratios of total/effector T-cells (50) and only $5 \%$ to $15 \%$ of tumors are PD-L1 positive with a $1 \%$ cut-off $(51,52)$, contrasting with high TILs and stromal macrophages PD-L1 expression. In addition, class I and II major histocompatibility complex (MHC) molecules and regulatory chemokines, such as IL-2 and Macrophage Activating Factor, are down-regulated in most SCLCs, contrasting with a high production of IL-15, all these findings favoring intrinsic resistance to immunotherapies (53).

Regarding epigenetic abnormalities, DNA methylation patterns can also define different SCLC subtypes. A global DNA hypomethylation is observed in most SCLCs, but a subgroup of SCLCs present hypermethylated promoter CPG islands leading to specific gene silencing. This subgroup is characterized by a poor prognosis and a high expression of the EZH2 (E2F target and histone methyltransferase) gene (41,54). EZH2 is a target of E2F, which is normally repressed by $\mathrm{RB} 1$, and overexpressed in SCLC due to the universal loss of RB1. EZH2 is a histone methyltransferase that forms the polycomb repressive complex 2 (PRC2) and plays a role in homeostasis of SCLC cells. EZH2 mediates tri-methylation of histone
$\mathrm{H} 3$ at lysine 27 (H3K27me3) in discrete promoter $\mathrm{CpG}$ islands, leading to transcriptional repression. Schlafen family member 11 (SLFN11) is one of the genes repressed by EZH2 and its loss of expression results in increased DNA damage repair after cytotoxic chemotherapy through Homologous Recombination system activation and this loss has been implicated in chemotherapy resistance in SCLC PDX (55). Other epigenetic abnormalities have been reported in SCLC, such as Caspase 8 (CASP8), FAS and TRAIL-R1 gene promoter silencing methylation (56).

\section{Therapeutic implications}

To date, SCLC standard of care consists in non-targeted approaches. They are based for limited stage SCLC on concurrent chemoradiation, and for extensive stage SCLC, on chemotherapy alone or chemotherapy combined with anti-PD-L1 checkpoint inhibitors, such as atezolizumab and durvalumab $(54,55)$. The most commonly recommended chemotherapy regimen is platinum-etoposide (PE), which has been proven to increase survival with less toxicity than other regimens that combine anthracyclines, vinca-alkaloids, methotrexate and/or cyclophosphamide (57). SCLCs are chemosensitive in first-line setting, with response rates ranging from $70 \%$ to $80 \%$, with up to $50 \%$ of complete responses. However, the majority of patients die from recurrences, which are refractory to chemotherapy. With the addition of atezolizumab to chemotherapy (58), median PFS has been shown to be 5.2 vs. 4.3 months $(\mathrm{HR}=0.77$, 95\% CI: 0.62-0.096, $\mathrm{P}=0.02$ ), and median OS reached 12.3 vs. 10.3 months (HR $=0.70,95 \%$ CI: $0.54-0.91, \mathrm{P}=0.007$ ). Interestingly, benefits were consistent across all patients' subgroups and no biomarker, including PD-L1 expression or tumor mutation load, predicted the magnitude of benefit in this trial, despite preliminary data suggesting some predictive role (59). Recurrent SCLC is divided into two categories: refractory (disease progression occurring less than 3 months from completion of initial therapy) or sensitive (progression after 3 months) (60). Rates of response to second-line therapy are substantially lower in patients with refractory/resistant disease. Reinitiation of the front-line chemotherapy regimen is proposed if the initial response duration is 6 months or more based on ported response rates of $50-60 \%$. The benefit of secondline chemotherapy in recurrent SCLC was evaluated in a randomized trial comparing oral topotecan with best supportive care (61). Although topotecan induced response in only $7 \%$ of patients, it did significantly improve overall 
survival.

With the recognition of SCLC variants, emerging therapies guided on biology could be proposed in the future. DLL3 inhibitors, including antibody drug conjugate, bi-specific $\mathrm{T}$ cell engager and chimeric antigen receptor (CAR)-T cell constructs, could be specifically recommended in SCLC-A (62). Rovalpituzumab teserine (Rova-T) is an antibody drug conjugate consisting of a monoclonal antibody targeting DLL3, a cathepsincleavable linker, and a pyrrolobenzodiazepine (PBD) warhead (62). The first-in-human clinical trial of Rova-T in recurrent SCLC demonstrated encouraging activity despite frequent severe side-effects attributable to the PBD warhead. However, subsequent studies including the phase 2 TRINITY study and the phase 3 TAHOE trial in the second line setting showed disappointing efficacy data leading to the discontinuation of the development of Rova-T $(63,64)$. Besides antibody-drug conjugates, other DLL3-targeting therapies are under investigation in SCLC and include the bispecific T cell engager (BiTE) AMG 757 (NCT03319940), and a chimeric antigen receptor CAR-T AMG119 (NCT03392064). Other strategies for SCLC-A could include BCL2-inhibitors, as BCL2 is a target of ASCL1, but limited clinical evidence is available in SCLC patients treated with BCL2 inhibitors such as venetoclax (65). Inhibitors of LSD1 (Lysine-specific histone demethylase A1), a flavin adenine dinucleotide FADdependent demethylase highly expressed in SCLC-A (66), are also in early stage development. The SCLC-N variant expressing high levels of MYC and NEUROD1 could be sensitive to Aurora kinase inhibitors when combined with chemotherapy as shown in mouse models (37). Singleagent alisertib (MLN8237) was evaluated in a phase 1 study which included 48 patients with SCLC, 36 presenting with chemotherapy-sensitive disease and the remaining with chemotherapy-refractory disease. The ORR in this group was $21 \%$ (67). Aurora amplification has also been associated with resistance to taxanes, justifying proposing a combination of paclitaxel as second line therapy. In a randomized phase II trial, the combination of alisertib plus paclitaxel showed a modest improvement in survival, with no statistical significance. Ultimately, as Aurora kinase also phosphorylates and impairs LKB1/STK11, whose loss is one of the resistance factors to immunotherapies, immune checkpoint inhibitors could benefit from the association with Aurora kinase inhibitors. Trials are ongoing with other agents such as LY3295668 that preclinically demonstrated synthetic lethal activity in RB1-deficient tumors (68).
Another strategy in SCLC-N could involve PI3K/mTOR pathway and HSP90 inhibitors (65). SCLC-P cell lines were reported to be sensitive to IGFR1-inhibitors (46), however, such inhibitors are currently not available in the clinic. SCLC-IM may actually represent the molecular subtype that would be biologically the most prone to be sensitive to immunotherapy, but this has not been formally tested so far. Ultimately, as PARP1 is highly expressed in SCLC, PARP inhibitors (PARPIs) were evaluated in combination with chemotherapy. A phase II trial with veliparib plus temozolomide in previously treated SCLC patients failed to meet its primary end-point of improved PFS but did show an improved ORR of $39 \%$. SLFN11 was a clear predictive marker of benefit with improved PFS (5.7 vs. 3.6 months) and OS (12.1 vs. 7.5 months) in the subset of patients with tissue for analysis (69). Veliparib was tested in combination with cisplatin and etoposide doublet in 128 patients with ES SCLC in a randomized control trial. The median PFS was 6.1 vs. 5.5 months [unstratified hazard ratio $(\mathrm{HR})=0.75$, one-sided $\mathrm{P}=0.06$ ] (70). PARPIs are also being studied in combination with various other novel agents, including the vascular endothelial growth factor inhibitor cediranib, or the WEE1 G2 checkpoint kinase inhibitor AZD1775 $(71,72)$. Figure 1 summarizes the main molecular subtypes of SCLC and LCNEC and the potential therapeutic impacts of such classification. However, despite all these encouraging perspectives for a class of tumors that until now has not benefited from targeted therapies, a major pitfall is the absence of selection of patients based on molecularlydefined SCLC subsets. Retrospective biomarker analyses are planned in some of the trials, but they are limited by the reduced availability of material from patients with advanced disease.

\section{LCNEC}

\section{Histopathological classification}

LCNEC is a high-grade neuroendocrine carcinoma with strong similarities to SCLC, regarding epidemiology, prognosis and genetics. LCNEC was classified in the 1999 and $2004 \mathrm{WHO}$ classifications of lung tumors as a largecell carcinoma variant $(5,73)$, but in the 2015 classification, LCNEC was in a separate chapter, close to SCLC (4). Its definition is based on the recognition of NE morphology and expression of NE markers by immunohistochemistry, or presence of NE granules with electron microscopy, which is rarely performed nowadays. However, large-cell carcinomas 

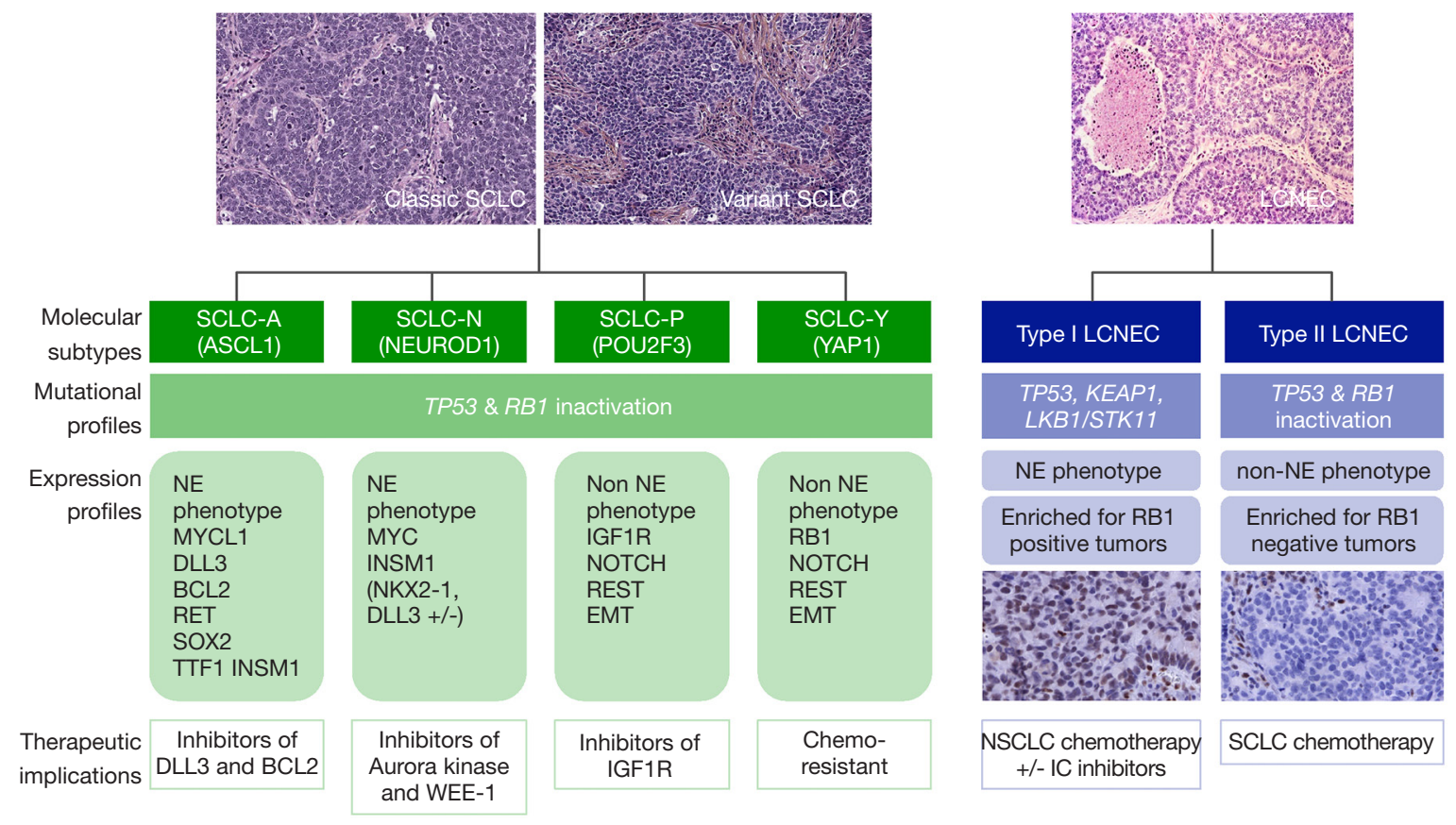

Figure 1 Main molecular subtypes of small cell lung carcinoma (SCLC) and large cell neuroendocrine carcinoma (LCNEC) with their potential therapeutic impacts. This figure includes cases of SCLC and LCNEC stained with Hematoxylin Eosin Saffron $(\times 200$ for SCLC and $\times 100$ for LCNEC), and LCNECs stained with RB1 antibody (immunohistochemistry using 13A10 clone from Leica Biosystems, Buffalo Grove, IL USA; immunoperoxidase, $\times 200$ ).

with NE morphology but non-NE immunophenotype have been reported, as well as large-cell carcinoma with non-NE morphology but with NE immunophenotype or ultrastructural features (17). To date, no preneoplastic lesions have been identified for high-grade NENs, but genomic abnormalities characteristic of these carcinomas has been detected in normal epithelial cells in smokers (74).

Histologically, LCNECs are characterized by a NE morphology with organoid nesting, trabecular growth, palisading or rosette-like structures; they typically exhibit a high mitotic rate, higher than 10 mitoses per $2 \mathrm{~mm}^{2}$, often between 80 and 100 per $2 \mathrm{~mm}^{2}$, and a non-small cell cytology with a cell size larger than three times the diameter of resting lymphocytes. However, some LCNECs presenting a predominant number of smaller cells have been reported, suggesting a significant size variability (75). Cytoplasms are classically ample and eosinophilic to basophilic, and there is a low nuclear-to-cytoplasm ratio. Chromatin is frequently vesicular with prominent nucleoli. Necrosis is often widespread but can be focal. All these diagnostic criteria have been described from surgical specimens, but they can be difficult to demonstrate on small biopsy specimens, as NE morphology is required for the diagnosis. Most of the time, these tumors are classified as non-small lung carcinomas on biopsies and cytology, and recognized as LCNEC only on subsequent surgical specimens. Even in these cases, recognition of $\mathrm{NE}$ morphology with palisading and rosettes can be subtle. Demonstration of NE markers, which is mandatory for the diagnosis of LCNEC, is of great help on small specimens. Among them, CD56 is probably the most sensitive for LCNEC diagnosis (expressed in $92 \%$ to $98 \%$ of LCNECs) but lacks specificity as nearly $10 \%$ of adenocarcinomas, squamous cell carcinomas, and large-cell carcinomas express it with up to $30 \%$ of positive cells. Conversely, chromogranin A, expressed in nearly $70 \%$ of LCNECs, is likely the most specific but lacks sensitivity. Synaptophysin is expressed in $87 \%$ LCNECs but lacks specificity as it can be found in up to $10 \%$ of adenocarcinomas and $5 \%$ of squamous cell carcinomas (76) but CD56, synaptophysin and chromogranin A are co-expressed in less than $4 \%$ of non-NE NSCLC $(77,78)$. The expression of these markers is widely dependent on the level of differentiation and at least $50 \%$ of malignant cells positive for one of the abovementioned antibodies is required for the diagnosis $(4,5)$. ASCL1 can also be detected in most LCNECs $(22,79)$, 
as well as TTF1 which is expressed by $41 \%$ to $75 \%$ of LCNECs (80-82).

\section{Molecular classification}

George et al. have provided a very comprehensive multiomics analysis of LCNECs based on whole exome/genome sequencing (WES/WGS) of 60 matched tumor-normal cases, RNA sequencing of 69 tumors, and Affymetrix 6.0 SNP arrays for copy number variations on 60 tumors (83). This study revealed amplifications of the $8 \mathrm{p} 12$ region containing the FGFR1 gene $(7 \%)$ and of the $14 \mathrm{q} 13$ region containing the NKX2-1 (TTF-1) gene (10\%), as in adenocarcinomas and squamous cell carcinomas of the lung, respectively, but also amplifications of the $1 \mathrm{p} 34$ region containing the MYCL1 gene (12\%) and of the $8 \mathrm{q} 24.21$ region containing the $M Y C$ gene (5\%), as in SCLC (see above). TP53 and RB1 mutations were found in $92 \%$ and $42 \%$ of the cases, respectively, as well as LKB1 (STK11) $(30 \%)$ and KEAP1 (22\%) mutations. In addition, lossof-heterozygosity (LOH), biallelic alterations and larger genomic rearrangements leading to somatic alterations of $R B 1$ and $S T K 11 / K E A P 1$ were found in up to $82 \%$ of the cases and were mutually exclusive. RAS-pathway (KRAS/ NRAS/HRAS) mutations (10\%) and BRAF mutations, which occur preferentially in lung adenocarcinomas, were also reported, as well as other genomic alterations affecting CREBBP, EP300, NOTCH, MEN1, and ARID1A. Subsequently, other studies using targeted sequencing (54,84-88) confirmed these observations, and found additional amplifications of $M Y C N(2 \%), S R Y-b o x 2$ (SOX2) (11\%), and cyclin E1 (CCNE1) (9\%) (85), as well as SMARCA2 mutations (11\%), PI3KCA-AKT-mTOR mutations (88), and neurotrophic receptor tyrosine kinase 2 (NTRK2) and 3 (NTRK3) gene mutations (19\%) (85).

Regarding expression profiles, LCNECs constitute their own class, differing from other NSCLCs and carcinoids, but close to SCLCs (83). They can be divided molecularly into two separate subtypes, the type I being characterized by STK11/KEAP1 alterations, but with an NE phenotype, high expression of ASCL1 and DLL3 and downregulation of NOTCH pathway, as in the SCLC classical subtype (see above). Conversely, the type II is characterized by RB1 alterations, but a predominant non-NE phenotype (with low expression of chromogranin A and synaptophysin), high levels of REST and NOTCH, and immune cell response activation. Rekhtman et al. (85) also deciphered different molecular subtypes: one with TP53/RB1 inactivation and
MYCL amplification, and another one with retained TP53/ RB1 functions, NOTCH mutations and either STK11/ KRAS/TTF1 mutations, similar to that of adenocarcinoma, or KEAP1 mutations or SOX2/FGFR1 amplifications, as with squamous cell carcinoma. Ultimately, they reported a "carcinoid-like" subtype with MEN1 mutations and low mutational burden. Gene expression profiling showed that SCLCs and LCNECs belong to the same clusters, and as LCNECs can be combined with SCLCs, suggesting that LCNECs probably originate from the same precursors than SCLCs. However, given their low ASCL1 expression and their higher expression of the non-NE specific marker hairy/enhancer of split 1 (HES1), a basic helix-loophelix transcription factor acting as a key effector of notch signaling pathway (89), LCNECs are also close to non-NE NSCLCs in some ways. Regarding targetable mutations, they are rarely detected in LCNECs, with to $2 \%$ and $1 \%$ of EGFR and BRAF mutations respectively, which are more frequently observed in wild-type RB1 LCNECs (85). By contrast, KRAS mutations are observed in $22-24 \%$ of LCNECs and are considered to be responsible for poor response to chemotherapy $(85,90)$.

\section{Therapeutic implications}

Surgical removal has to be considered each time it is possible and a perioperative chemotherapy has been suggested to be beneficial to patients with resected LCNEC $(91,92)$. For advanced stages, there is no standard of treatment for LCNEC, which can be either treated by SCLC-type chemotherapy (platinum-etoposide based) (93-99) or by NSCLC-type chemotherapy regimens (gemcitabine/taxane/pemetrexed combined with platinum). However, LCNEC seemed to respond poorly to PEbased chemotherapy (100), with a far better benefit obtained with gemcitabine- or taxane-based chemotherapy $(87,90,99,101,102)$. Subsequently Derks JL and al showed in a retrospective series of LCNECs classified according to their molecular profile that patients with LCNECs RB1 wild-type and/or expressing RB1 by immunohistochemistry have superior overall survival when treated with NSCLClike chemotherapy compared to SCLC-like chemotherapy (9.6 vs. 5.6 months) (87), with no difference in outcome for patients with inactivated $R B 1$. The effectiveness of secondline chemotherapy could be different between LCNECs and SCLCs (103). While PD1/PDL1 inhibitors showed no efficacy in SCLCs as a second-line treatment, responses are reported in LCNECs in late-line setting with overall 
similar efficacy as in NSCLCs $(104,105)$. Trials are ongoing to further confirm this finding (NCT03591731) (Figure 1). Ultimately, LCNECs which may harbor targetable abnormalities should be systematically screened to drive potential access to precision medicine approaches using kinase inhibitors.

\section{Conclusions}

The above summarized genomic studies on SCLCs and LCNECs have provided new potential avenues for a more adapted treatment of these aggressive cancers. Although promising, the effectiveness of these therapeutic options needs extensive and rigorous testing in thoroughly designed clinical trials before reaching the clinical setting.

\section{Acknowledgments}

We want to thank Drs Bregtje Hermans and Jules Derks from the Maastricht University, Netherlands, for providing us with the RB1 immunohistochemistry pictures.

Funding: None.

\section{Footnote}

Provenance and Peer Review: This article was commissioned by the Guest Editor (Helmut H. Popper) for the series "New Developments in Lung Cancer Diagnosis and Pathological Patient Management Strategies" published in Translational Lung Cancer Research. The article was sent for external peer review organized by the Guest Editor and the editorial office.

Conflicts of Interest: All authors have completed the ICMJE uniform disclosure form (available at http://dx.doi. org/10.21037/tlcr-20-269). The series "New Developments in Lung Cancer Diagnosis and Pathological Patient Management Strategies" was commissioned by the editorial office without any funding or sponsorship. The authors have no other conflicts of interest to declare.

Ethical Statement: The authors are accountable for all aspects of the work in ensuring that questions related to the accuracy or integrity of any part of the work are appropriately investigated and resolved.

Disclaimer: Where authors are identified as personnel of the International Agency for Research on Cancer/World
Health Organization, the authors alone are responsible for the views expressed in this article and they do not necessarily represent the decisions, policy or views of the International Agency for Research on Cancer/World Health Organization.

Open Access Statement: This is an Open Access article distributed in accordance with the Creative Commons Attribution-NonCommercial-NoDerivs 4.0 International License (CC BY-NC-ND 4.0), which permits the noncommercial replication and distribution of the article with the strict proviso that no changes or edits are made and the original work is properly cited (including links to both the formal publication through the relevant DOI and the license). See: https://creativecommons.org/licenses/by-nc-nd/4.0/.

\section{References}

1. Derks JL, Hendriks LE, Buikhuisen WA, et al. Clinical features of large cell neuroendocrine carcinoma: a population-based overview. Eur Respir J 2016;47:615-24.

2. Govindan R, Page N, Morgensztern D, et al. Changing epidemiology of small-cell lung cancer in the United States over the last 30 years: analysis of the surveillance, epidemiologic, and end results database. J Clin Oncol 2006;24:4539-44.

3. Travis WD, Brambilla E, Nicholson AG, et al. The 2015 World Health Organization Classification of Lung Tumors: Impact of Genetic, Clinical and Radiologic Advances Since the 2004 Classification. J Thorac Oncol 2015;10:1243-60.

4. Travis WD, Brambilla E, Burke AP, et al. WHO Classification of Tumours of the Lung, Pleura, Thymus and Heart. 4th Ed. Geneva: WHO, 2015.

5. Travis WD, Brambilla E, Müller-Hermelink HK, et al. eds. Pathology and Genetics of Tumours of the Lung, Pleura, Thymus and Heart. Lyon: IARC Press, 2004.

6. Vollmer RT. The effect of cell size on the pathologic diagnosis of small and large cell carcinomas of the lung. Cancer 1982;50:1380-3.

7. Nicholson SA, Beasley MB, Brambilla E, et al. Small cell lung carcinoma (SCLC): a clinicopathologic study of 100 cases with surgical specimens. Am J Surg Pathol 2002;26:1184-97.

8. Ruffini E, Rena O, Oliaro A, et al. Lung tumors with mixed histologic pattern. Clinico-pathologic characteristics and prognostic significance. Eur J Cardiothorac Surg 2002;22:701-7. 
9. Travis WD. Pathology of lung cancer. Clin Chest Med 2002;23:65-81, viii.

10. World Health Organization. Histological Typing of Lung Tumours. 2nd Ed. Geneva: World Health Organization, 1981.

11. Gazdar AF, Carney DN, Nau MM, et al. Characterization of variant subclasses of cell lines derived from small cell lung cancer having distinctive biochemical, morphological, and growth properties. Cancer Res 1985;45:2924-30.

12. Miki M, Ball DW, Linnoila RI. Insights into the achaetescute homolog-1 gene (hASH1) in normal and neoplastic human lung. Lung Cancer 2012;75:58-65.

13. Ye B, Cappel J, Findeis-Hosey J, et al. hASH1 is a specific immunohistochemical marker for lung neuroendocrine tumors. Hum Pathol 2016;48:142-7.

14. Thunnissen E, Borczuk AC, Flieder DB, et al. The Use of Immunohistochemistry Improves the Diagnosis of Small Cell Lung Cancer and Its Differential Diagnosis. An International Reproducibility Study in a Demanding Set of Cases. J Thorac Oncol 2017;12:334-46.

15. Lantuejoul S, Moro D, Michalides RJ, et al. Neural cell adhesion molecules (NCAM) and NCAM-PSA expression in neuroendocrine lung tumors. Am J Surg Pathol 1998;22:1267-76.

16. Hasleton PS, Flieder DB. editors. Spencer's Pathology of the Lung. 6th ed. Cambridge: Cambridge University Press, 2013.

17. Travis WD. Advances in neuroendocrine lung tumors. Ann Oncol 2010;21 Suppl 7:vii65-71.

18. Caplin ME, Baudin E, Ferolla P, et al. Pulmonary neuroendocrine (carcinoid) tumors: European Neuroendocrine Tumor Society expert consensus and recommendations for best practice for typical and atypical pulmonary carcinoids. Ann Oncol 2015;26:1604-20.

19. Wick MR, Marchevsky AM. Neuroendocrine neoplasms of the lung: Concepts and terminology. Semin Diagn Pathol 2015;32:445-55.

20. Kontogianni K, Nicholson AG, Butcher D, et al. CD56: a useful tool for the diagnosis of small cell lung carcinomas on biopsies with extensive crush artefact. J Clin Pathol 2005;58:978-80.

21. Guinee DG, Fishback NF, Koss MN, et al. The spectrum of immunohistochemical staining of small-cell lung carcinoma in specimens from transbronchial and openlung biopsies. Am J Clin Pathol 1994;102:406-14.

22. Hiroshima K, Iyoda A, Shida T, et al. Distinction of pulmonary large cell neuroendocrine carcinoma from small cell lung carcinoma: a morphological, immunohistochemical, and molecular analysis. Mod Pathol 2006;19:1358-68.

23. Leversha MA, Fielding P, Watson S, et al. Expression of p53, pRB, and p16 in lung tumours: a validation study on tissue microarrays. J Pathol 2003;200:610-9.

24. Boers JE, den Brok JL, Koudstaal J, et al. Number and proliferation of neuroendocrine cells in normal human airway epithelium. Am J Respir Crit Care Med 1996;154:758-63.

25. Sutherland KD, Proost N, Brouns I, et al. Cell of origin of small cell lung cancer: inactivation of Trp53 and Rb1 in distinct cell types of adult mouse lung. Cancer Cell 2011;19:754-64.

26. Oser MG, Niederst MJ, Sequist LV, et al. Transformation from non-small-cell lung cancer to small-cell lung cancer: molecular drivers and cells of origin. Lancet Oncol 2015;16:e165-72.

27. Lee JK, Lee J, Kim S, et al. Clonal History and Genetic Predictors of Transformation Into Small-Cell Carcinomas From Lung Adenocarcinomas. J Clin Oncol 2017;35:3065-74.

28. Lin C, Song H, Huang C, et al. Alveolar type II cells possess the capability of initiating lung tumor development. PLoS One 2012;7:e53817.

29. Kim DW, Wu N, Kim YC, et al. Genetic requirement for Mycl and efficacy of RNA Pol I inhibition in mouse models of small cell lung cancer. Genes Dev 2016;30:1289-99.

30. Peifer M, Fernández-Cuesta L, Sos ML, et al. Integrative genome analyses identify key somatic driver mutations of small-cell lung cancer. Nat Genet 2012;44:1104-10.

31. Rudin CM, Durinck S, Stawiski EW, et al. Comprehensive genomic analysis identifies SOX2 as a frequently amplified gene in small-cell lung cancer. Nat Genet 2012;44:1111-6.

32. George J, Lim JS, Jang SJ, et al. Comprehensive genomic profiles of small cell lung cancer. Nature 2015;524:47-53.

33. Ito T, Udaka N, Yazawa T, et al. Basic helix-loophelix transcription factors regulate the neuroendocrine differentiation of fetal mouse pulmonary epithelium. Development 2000;127:3913-21.

34. Horie M, Miyashita N, Mattsson JSM, et al. An integrative transcriptome analysis reveals a functional role for thyroid transcription factor- 1 in small cell lung cancer. J Pathol 2018;246:154-65.

35. Sakaeda M, Sato H, Ishii J, et al. Neural lineagespecific homeoprotein BRN2 is directly involved in TTF1 expression in small-cell lung cancer. Lab Invest 2013;93:408-21.

36. Borromeo MD, Savage TK, Kollipara RK, et al. ASCL1 
and NEUROD1 Reveal Heterogeneity in Pulmonary Neuroendocrine Tumors and Regulate Distinct Genetic Programs. Cell Rep 2016;16:1259-72.

37. Mollaoglu G, Guthrie MR, Böhm S, et al. MYC Drives Progression of Small Cell Lung Cancer to a Variant Neuroendocrine Subtype with Vulnerability to Aurora Kinase Inhibition. Cancer Cell 2017;31:270-85.

38. Poirier JT, Dobromilskaya I, Moriarty WF, et al. Selective tropism of Seneca Valley virus for variant subtype small cell lung cancer. J Natl Cancer Inst 2013;105:1059-65.

39. Carney DN, Gazdar AF, Bepler G, et al. Establishment and identification of small cell lung cancer cell lines having classic and variant features. Cancer Res 1985;45:2913-23.

40. Zhang W, Girard L, Zhang YA, et al. Small cell lung cancer tumors and preclinical models display heterogeneity of neuroendocrine phenotypes. Transl Lung Cancer Res 2018;7:32-49.

41. Poirier JT, Gardner EE, Connis N, et al. DNA methylation in small cell lung cancer defines distinct disease subtypes and correlates with high expression of EZH2. Oncogene 2015;34:5869-78.

42. Carney DN, Mitchell JB, Kinsella TJ. In vitro radiation and chemotherapy sensitivity of established cell lines of human small cell lung cancer and its large cell morphological variants. Cancer Res 1983;43:2806-11.

43. Lim JS, Ibaseta A, Fischer MM, et al. Intratumoural heterogeneity generated by Notch signalling promotes small-cell lung cancer. Nature 2017;545:360-4.

44. Hassan WA, Yoshida R, Kudoh S, et al. Notch1 controls cell chemoresistance in small cell lung carcinoma cells. Thorac Cancer 2016;7:123-8.

45. McColl K, Wildey G, Sakre N, et al. Reciprocal expression of INSM1 and YAP1 defines subgroups in small cell lung cancer. Oncotarget 2017;8:73745-56.

46. Huang $\mathrm{YH}, \mathrm{Klingbeil} \mathrm{O}, \mathrm{He} \mathrm{XY}$, et al. POU2F3 is a master regulator of a tuft cell-like variant of small cell lung cancer. Genes Dev 2018;32:915-28.

47. Rudin CM, Poirier JT, Byers LA, et al. Molecular subtypes of small cell lung cancer: a synthesis of human and mouse model data. Nat Rev Cancer 2019;19:289-97.

48. Sonkin D, Thomas A, Teicher BA. Are neuroendocrine negative small cell lung cancer and large cell neuroendocrine carcinoma with WT RB1 two faces of the same entity? Lung Cancer Manag 2019;8:LMT13.

49. Gay CM, Diao L, Stewart CA, et al. ASCL1, NEUROD1, and POU2F3 Drive Distinct Subtypes of Small Cell Lung Cancer with Unique Therapeutic Vulnerabilities. J Thorac Oncol 2019;14:S213.
50. Carvajal-Hausdorf D, Altan M, Velcheti V, et al. Expression and clinical significance of PD-L1, B7-H3, B7$\mathrm{H} 4$ and TILs in human small cell lung Cancer (SCLC). J Immunother Cancer 2019;7:65.

51. Yu H, Batenchuk C, Badzio A, et al. PD-L1 Expression by Two Complementary Diagnostic Assays and mRNA In Situ Hybridization in Small Cell Lung Cancer. J Thorac Oncol 2017;12:110-20.

52. Schultheis AM, Scheel AH, Ozretić L, et al. PD-L1 expression in small cell neuroendocrine carcinomas. Eur J Cancer 2015;51:421-6.

53. Tian $\mathrm{Y}$, Zhai X, Han A, et al. Potential immune escape mechanisms underlying the distinct clinical outcome of immune checkpoint blockades in small cell lung cancer. J Hematol Oncol 2019;12:67.

54. Karlsson A, Jönsson M, Lauss M, et al. Genome-wide DNA methylation analysis of lung carcinoma reveals one neuroendocrine and four adenocarcinoma epitypes associated with patient outcome. Clin Cancer Res 2014;20:6127-40.

55. Gardner EE, Lok BH, Schneeberger VE, et al. Chemosensitive Relapse in Small Cell Lung Cancer Proceeds through an EZH2-SLFN11 Axis. Cancer Cell 2017;31:286-99.

56. Hopkins-Donaldson S, Cathomas R, Simões-Wüst AP, et al. Induction of apoptosis and chemosensitization of mesothelioma cells by Bcl-2 and Bcl-xL antisense treatment. Int J Cancer 2003;106:160-6.

57. Available online: https://www.nccn.org/professionals/ physician_gls/pdf/sclc.pdf

58. Horn L, Mansfield AS, Szczęsna A, et al. First-Line Atezolizumab plus Chemotherapy in Extensive-Stage Small-Cell Lung Cancer. N Engl J Med 2018;379:2220-9.

59. Antonia SJ, López-Martin JA, Bendell J, et al. Nivolumab alone and nivolumab plus ipilimumab in recurrent smallcell lung cancer (CheckMate 032): a multicentre, openlabel, phase 1/2 trial. Lancet Oncol 2016;17:883-95.

60. Gazdar AF, Bunn PA, Minna JD. Small-cell lung cancer: what we know, what we need to know and the path forward. Nat Rev Cancer 2017;17:765.

61. Pelayo Alvarez M, Westeel V, et al. Chemotherapy versus best supportive care for extensive small cell lung cancer. Cochrane Database Syst Rev 2013;(11):CD001990.

62. Saunders LR, Bankovich AJ, Anderson WC, et al. A DLL3-targeted antibody-drug conjugate eradicates highgrade pulmonary neuroendocrine tumor-initiating cells in vivo. Sci Transl Med 2015;7:302ra136.

63. Morgensztern D, Besse B, Greillier L, et al. Efficacy 
and Safety of Rovalpituzumab Tesirine in ThirdLine and Beyond Patients with DLL3-Expressing, Relapsed/Refractory Small-Cell Lung Cancer: Results From the Phase II TRINITY Study. Clin Cancer Res 2019;25:6958-66.

64. Available online: https://news.abbvie.com/news/phase-3trial-rova-t-as-second-line-therapy-for-advanced-smallcell-lung-cancer-tahoe-study-halted.htm

65. Cardnell RJ, Li L, Sen T, et al. Protein expression of TTF1 and cMYC define distinct molecular subgroups of small cell lung cancer with unique vulnerabilities to aurora kinase inhibition, DLL3 targeting, and other targeted therapies. Oncotarget 2017;8:73419-32.

66. Augert A, Eastwood E, Ibrahim AH, et al. Targeting NOTCH activation in small cell lung cancer through LSD1 inhibition. Sci Signal 2019;12:eaau2922.

67. Melichar B, Adenis A, Lockhart AC, et al. Safety and activity of alisertib, an investigational aurora kinase A inhibitor, in patients with breast cancer, small-cell lung cancer, non-small-cell lung cancer, head and neck squamous-cell carcinoma, and gastro-oesophageal adenocarcinoma: a five-arm phase 2 study. Lancet Oncol 2015;16:395-405.

68. Gong X, Du J, Parsons SH, et al. Aurora A Kinase Inhibition Is Synthetic Lethal with Loss of the RB1 Tumor Suppressor Gene. Cancer Discov 2019;9:248-63.

69. Byers LA, Krug L, Waqar S, et al. MA11.07. Improved small cell lung cancer (SCLC) response rates with veliparib and temozolomide: results from a phase II trial. J Thorac Oncol 2017;12:S406-7.

70. Owonikoko TK, Dahlberg SE, Sica GL, et al. Randomized Phase II Trial of Cisplatin and Etoposide in Combination With Veliparib or Placebo for Extensive-Stage Small-Cell Lung Cancer: ECOG-ACRIN 2511 Study. J Clin Oncol 2019;37:222-9.

71. Liu JF, Barry WT, Birrer M, et al. Combination cediranib and olaparib versus olaparib alone for women with recurrent platinum-sensitive ovarian cancer: a randomised phase 2 study. Lancet Oncol 2014;15:1207-14.

72. Lallo A, Frese KK, Morrow CJ, et al. The Combination of the PARP Inhibitor Olaparib and the WEE1 Inhibitor AZD1775 as a New Therapeutic Option for Small Cell Lung Cancer. Clin Cancer Res 2018;24:5153-64.

73. Travis WD, Colby TV, Corrin B, et al. Sobin and pathologists from 14 countries. Histological Typing of Lung and Pleural Tumors. 3rd ed. Berlin: Springer, 1999.

74. Swarts DR, Ramaekers FC, Speel EJ. Molecular and cellular biology of neuroendocrine lung tumors: evidence for separate biological entities. Biochim Biophys Acta 2012;1826:255-71.

75. Marchevsky AM, Gal AA, Shah S, et al. Morphometry confirms the presence of considerable nuclear size overlap between "small cells" and "large cells" in high-grade pulmonary neuroendocrine neoplasms. Am J Clin Pathol 2001;116:466-72.

76. Ionescu DN, Treaba D, Gilks CB, et al. Nonsmall cell lung carcinoma with neuroendocrine differentiation--an entity of no clinical or prognostic significance. Am J Surg Pathol 2007;31:26-32.

77. Sterlacci W, Fiegl M, Hilbe W, et al. Clinical relevance of neuroendocrine differentiation in non-small cell lung cancer assessed by immunohistochemistry: a retrospective study on 405 surgically resected cases. Virchows Arch 2009;455:125-32.

78. Derks JL, Dingemans AC, van Suylen RJ, et al. Is the sum of positive neuroendocrine immunohistochemical stains useful for diagnosis of large cell neuroendocrine carcinoma (LCNEC) on biopsy specimens? Histopathology 2019;74:555-66.

79. Borges M, Linnoila RI, van de Velde HJ, et al. An achaete-scute homologue essential for neuroendocrine differentiation in the lung. Nature 1997;386:852-5.

80. Sturm N, Lantuéjoul S, Laverrière $\mathrm{MH}$, et al. Thyroid transcription factor 1 and cytokeratins 1, 5, 10, 14 (34betaE12) expression in basaloid and large-cell neuroendocrine carcinomas of the lung. Hum Pathol 2001;32:918-25.

81. Du EZ, Goldstraw P, Zacharias J, et al. TTF-1 expression is specific for lung primary in typical and atypical carcinoids: TTF-1-positive carcinoids are predominantly in peripheral location. Hum Pathol 2004;35:825-31.

82. Carlson JW, Nucci MR, Brodsky J, et al. Biomarkerassisted diagnosis of ovarian, cervical and pulmonary small cell carcinomas: the role of TTF-1, WT-1 and HPV analysis. Histopathology 2007;51:305-12.

83. George J, Walter V, Peifer M, et al. Integrative genomic profiling of large-cell neuroendocrine carcinomas reveals distinct subtypes of high-grade neuroendocrine lung tumors. Nat Commun 2018;9:1048.

84. Vollbrecht C, Werner R, Walter RFH, et al. Mutational analysis of pulmonary tumours with neuroendocrine features using targeted massive parallel sequencing: a comparison of a neglected tumour group. Br J Cancer 2015;113:1704-11.

85. Rekhtman N, Pietanza MC, Hellmann MD, et al. Next-Generation Sequencing of Pulmonary Large 
Cell Neuroendocrine Carcinoma Reveals Small Cell

Carcinoma-like and Non-Small Cell Carcinoma-like

Subsets. Clin Cancer Res 2016;22:3618-29.

86. Miyoshi T, Umemura S, Matsumura Y, et al. Genomic Profiling of Large-Cell Neuroendocrine Carcinoma of the Lung. Clin Cancer Res 2017;23:757-65.

87. Derks JL, Leblay N, Thunnissen E, et al. Molecular Subtypes of Pulmonary Large-cell Neuroendocrine Carcinoma Predict Chemotherapy Treatment Outcome. Clin Cancer Res 2018;24:33-42.

88. Simbolo M, Barbi S, Fassan M, et al. Gene Expression Profiling of Lung Atypical Carcinoids and Large Cell Neuroendocrine Carcinomas Identifies Three Transcriptomic Subtypes with Specific Genomic Alterations. J Thorac Oncol 2019;14:1651-61.

89. Nasgashio R, Sato Y, Matsumoto T, et al. The balance between the expressions of hASH1 and HES1 differs between large cell neuroendocrine carcinoma and small cell carcinoma of the lung. Lung Cancer 2011;74:405-10.

90. Naidoo J, Santos-Zabala ML, Iyriboz T, et al. Large Cell Neuroendocrine Carcinoma of the Lung: ClinicoPathologic Features, Treatment, and Outcomes. Clin Lung Cancer 2016;17:e121-9.

91. Abedallaa N, Tremblay L, Baey C, et al. Effect of chemotherapy in patients with resected small-cell or large-cell neuroendocrine carcinoma. J Thorac Oncol 2012;7:1179-83.

92. Sarkaria IS, Iyoda A, Roh MS, et al. Neoadjuvant and adjuvant chemotherapy in resected pulmonary large cell neuroendocrine carcinomas: a single institution experience. Ann Thorac Surg 2011;92:1180-6; discussion 1186-7.

93. Iyoda A, Hiroshima K, Moriya Y, et al. Prospective study of adjuvant chemotherapy for pulmonary large cell neuroendocrine carcinoma. Ann Thorac Surg 2006;82:1802-7.

94. Rossi G, Cavazza A, Marchioni A, et al. Role of chemotherapy and the receptor tyrosine kinases KIT, PDGFRalpha, PDGFRbeta, and Met in large-cell neuroendocrine carcinoma of the lung. J Clin Oncol 2005;23:8774-85.

95. Yamazaki S, Sekine I, Matsuno Y, et al. Clinical responses of large cell neuroendocrine carcinoma of the lung to cisplatin-based chemotherapy. Lung Cancer 2005;49:217-23.

96. Veronesi G, Morandi U, Alloisio M, et al. Large cell neuroendocrine carcinoma of the lung: a retrospective analysis of 144 surgical cases. Lung Cancer 2006;53:111-5.

97. Le Treut J, Sault MC, Lena H, et al. Multicentre phase II study of cisplatin-etoposide chemotherapy for advanced large-cell neuroendocrine lung carcinoma: the GFPC 0302 study. Ann Oncol 2013;24:1548-52.

98. Sun JM, Ahn MJ, Ahn JS, et al. Chemotherapy for pulmonary large cell neuroendocrine carcinoma: similar to that for small cell lung cancer or non-small cell lung cancer? Lung Cancer 2012;77:365-70.

99. Masters GA, Temin S, Azzoli CG, et al. Systemic Therapy for Stage IV Non-Small-Cell Lung Cancer: American Society of Clinical Oncology Clinical Practice Guideline Update. J Clin Oncol 2015;33:3488-515.

100. Dowlati A, Lipka MB, McColl K, et al. Clinical correlation of extensive-stage small-cell lung cancer genomics. Ann Oncol 2016;27:642-7.

101. Christopoulos P, Engel-Riedel W, Grohé C, et al. Everolimus with paclitaxel and carboplatin as firstline treatment for metastatic large-cell neuroendocrine lung carcinoma: a multicenter phase II trial. Ann Oncol 2017;28:1898-902.

102. Niho S, Kenmotsu H, Sekine I, et al. Combination chemotherapy with irinotecan and cisplatin for large-cell neuroendocrine carcinoma of the lung: a multicenter phase II study. J Thorac Oncol 2013;8:980-4.

103. Shimada Y, Niho S, Ishii G, et al. Clinical features of unresectable high-grade lung neuroendocrine carcinoma diagnosed using biopsy specimens. Lung Cancer 2012;75:368-73.

104. Mauclet C, Duplaquet F, Pirard L, et al. Complete tumor response of a locally advanced lung large-cell neuroendocrine carcinoma after palliative thoracic radiotherapy and immunotherapy with nivolumab. Lung Cancer 2019;128:53-6.

105. Chauhan A, Arnold SM, Kolesar J, et al. Immune checkpoint inhibitors in large cell neuroendocrine carcinoma: current status. Oncotarget 2018;9:14738-40.

Cite this article as: Lantuejoul S, Fernandez-Cuesta L, Damiola F, Girard N, McLeer A. New molecular classification of large cell neuroendocrine carcinoma and small cell lung carcinoma with potential therapeutic impacts. Transl Lung Cancer Res 2020;9(5):2233-2244. doi: 10.21037/tlcr-20-269 